Tadeusz Poreda, Institute of Mathematics, Technical University of Łódź, Łódź, Poland. email: poredat@p.lodz.pl

Wiesława Poreda, Faculty of Mathematics and Computer Science, University of Łódź, Łódź, Poland. email: poreda@math.uni.lodz.pl

\title{
ON THE SUMS OF TWO QUASI-CONTINUOUS FUNCTIONS WITH CLOSED GRAPHS
}

\begin{abstract}
In 2000 J. Borsík, J. Doboš, and M. Repický characterized sums of quasi-continuous functions with closed graphs. More precisely, they showed that such a sum must be Baire one star, and proved that each Baire one star function defined on a separable metric space which is Baire in the strong sense is the sum of three quasi-continuous functions with closed graphs. They showed also that not every Baire one star function defined on $\mathbb{R}$ is the sum of two quasi-continuous functions with closed graphs, and asked for characterization of such sums. The goal of this article is to present the required characterization.
\end{abstract}

\section{Preliminaries.}

Let $\mathbb{R}$ and $\mathbb{N}$ denote the real line and the set of all positive integers, respectively. The symbol $\omega_{1}$ denotes the first uncountable ordinal.

Throughout the paper we consider a fixed separable metric space $(X, d)$ which is Baire in the strong sense; i.e., each closed subset of $X$ is a Baire space. Let $B(x, r)$ stand for the open ball with radius $r$ centered at $x$. If $x \in X$ and $A$ is a nonvoid subset of $X$, then we define

$$
\operatorname{dist}(x, A) \stackrel{\mathrm{df}}{=} \inf \{d(x, t): t \in A\} .
$$

Mathematical Reviews subject classification: Primary: 26A21, 54C30; Secondary: 26A15, $54 \mathrm{C} 08$

Key words: quasi-continuous functions, closed graph

Received by the editors March 23, 2009

Communicated by: Krzysztof Ciesielski 
If $A \subset X$, then the symbols $\operatorname{int} A$ and $\mathrm{cl} A$ denote the interior and the closure of $A$, respectively. The word function denotes a mapping from a subset of $X$ into $\mathbb{R}$. The symbol $\mathcal{C}_{f}$ stands for the set of points of continuity of a function $f$, and $\mathcal{D}_{f} \stackrel{\text { df }}{=} X \backslash \mathcal{C}_{f}$.

Let $f: X \rightarrow \mathbb{R}$. We say that $f$ is a Baire one star function [7] (see also [6]), if for each nonempty closed set $P \subset X$, there exists an open set $U \subset X$ such that $P \cap U \neq \emptyset$ and $f \uparrow_{P \cap U}$ is continuous. We say that $f$ is quasi-continuous in the sense of Kempisty (cf. [4]), if for each $x \in X$, each neighborhood $U$ of $x$ and each $\varepsilon>0$, there is a nonvoid open set $V \subset U$ such that $\operatorname{diam} f[V \cup\{x\}]<\varepsilon$.

J. Borsík proved in 2002 that each function with closed graph defined on a complete metric space is Baire one star [2]. Clearly the sum of Baire one star functions is Baire one star as well. Moreover each Baire one star function is the sum of two functions with closed graphs [2], and it is the sum of two quasicontinuous functions [1]. However, there are Baire one star functions defined on $\mathbb{R}$ which cannot be written as the sum of two quasi-continuous functions with closed graphs [3]. We shall prove the following theorem.

Theorem. Let $f: X \rightarrow \mathbb{R}$. The following conditions are equivalent:

1. there are quasi-continuous functions with closed graphs $f_{1}, f_{2}: X \rightarrow \mathbb{R}$ such that $f=f_{1}+f_{2}$ on $X$,

2. $f$ is Baire one star and for each $x \in X$,

$$
\limsup _{t \rightarrow x, t \in \mathcal{C}_{f}}|f(t)|=\infty \quad \text { or } \quad \liminf _{t \rightarrow x, t \in \mathcal{C}_{f}}|f(t)-f(x)|=0 .
$$

We shall divide the proof into two parts. The proof of necessity is quite simple, see below. The proof of sufficiency is postponed to the next section.

Proof of NECESSITY. Let $f_{1}, f_{2}: X \rightarrow \mathbb{R}$ be quasi-continuous functions with closed graphs such that $f=f_{1}+f_{2}$ on $X$. By [3], $f$ is Baire one star.

Fix an $x \in X$ such that

$$
\limsup _{t \rightarrow x, t \in \mathfrak{e}_{f}}|f(t)|<\infty .
$$

Since $f_{1}$ is a quasi-continuous function defined on a Baire space $X$, there is a sequence $\left(x_{n}\right) \subset \mathcal{C}_{f}$ such that $x_{n} \rightarrow x$ and $f_{1}\left(x_{n}\right) \rightarrow f_{1}(x)$. (Cf., e.g., [5].) By (2), the sequence $\left(f_{2}\left(x_{n}\right)\right)$ is bounded. So, it has a subsequence, say $\left(f_{2}\left(x_{n_{k}}\right)\right)$, convergent to some $y \in \mathbb{R}$. Since function $f_{2}$ has closed graph, 
then $y=f_{2}(x)$. Consequently,

$$
\begin{aligned}
\liminf _{t \rightarrow x, t \in \mathfrak{C}_{f}}|f(t)-f(x)| & \leq \lim _{k \rightarrow \infty}\left|f\left(x_{n_{k}}\right)-f(x)\right| \\
& \leq \lim _{k \rightarrow \infty}\left|f_{1}\left(x_{n_{k}}\right)-f_{1}(x)\right|+\lim _{k \rightarrow \infty}\left|f_{2}\left(x_{n_{k}}\right)-f_{2}(x)\right|=0 .
\end{aligned}
$$

\section{Proof of Sufficiency.}

First we define some transfinite sequence of closed subsets of $X$. Put

$$
F_{0} \stackrel{\text { df }}{=} \mathrm{cl} \mathcal{D}_{f} .
$$

Assume that we have already defined the closed sets $F_{\beta}$ for each $\beta<\alpha$, where $\alpha$ is some ordinal. If $\alpha=\gamma+1$ for some ordinal $\gamma$, then we put

$$
F_{\alpha} \stackrel{\mathrm{df}}{=} \operatorname{cl} \mathcal{D}_{f \uparrow_{F_{\gamma}}},
$$

and otherwise we let

$$
F_{\alpha} \stackrel{\text { df }}{=} \bigcap_{\beta<\alpha} F_{\beta} .
$$

It can be readily verified that $F_{\alpha_{1}} \supset F_{\alpha_{2}}$ whenever $\alpha_{1}<\alpha_{2}$. So, $F_{\xi}=F_{\xi+1}$ for some ordinal $\xi$.

Since $f$ is Baire one star, $F_{\alpha+1}$ is nowhere dense in $F_{\alpha}$ for each ordinal $\alpha$. Using the fact that $X$ is second countable, we conclude that there is an ordinal $\xi$ for which $F_{\xi}=\emptyset$, and the least such ordinal is countable.

Let $(\lambda, \varrho): \mathbb{N} \rightarrow \xi \times \mathbb{N}$ be any bijection. For brevity, for each closed set $A \subset X$, we define the function $h_{A}: X \backslash A \rightarrow[0, \infty)$ as follows:

$$
h_{A}(x) \stackrel{\mathrm{df}}{=} \begin{cases}\frac{1}{\operatorname{dist}(x, A)} & \text { if } A \text { is nonvoid, } \\ 0 & \text { otherwise. }\end{cases}
$$

For each $n \in \mathbb{N}$, we define the function $M_{n}: X \backslash F_{\lambda(n)+1} \rightarrow[0, \infty)$ by

$$
M_{n}(t) \stackrel{\mathrm{df}}{=}|f(t)|+n+h_{F_{\lambda(n)+1}}(t) .
$$

Moreover for each $n \in \mathbb{N}$ and each $t \in X \backslash F_{\lambda(n)+1}$, we define

$$
U_{n}(t) \stackrel{\mathrm{df}}{=}\left(f(t)-(2 n)^{-1}, f(t)+(2 n)^{-1}\right) \cup\left(-\infty,-2 M_{n}(t)\right) \cup\left(2 M_{n}(t), \infty\right) .
$$




\subsection{Families $\mathcal{L}_{\varrho(n)}^{\lambda(n)}$.}

For each $n \in \mathbb{N}$, we shall construct the family $\mathcal{L}_{\varrho(n)}^{\lambda(n)}$, consisting of pairwise disjoint open sets, so that the following conditions are satisfied:

(E1) $\operatorname{diam} K<(2 n)^{-1}$ for each $K \in \mathcal{L}_{\varrho(n)}^{\lambda(n)}$,

(E2) the set $E_{n} \stackrel{\text { df }}{=} \bigcup_{K \in \mathcal{L}_{\varrho(n)}^{\lambda(n)}} \mathrm{cl} K$ is a subset of $X \backslash F_{0}$ which is closed in $X$,

(E3) $E_{n} \subset \bigcup_{x \in F_{\lambda(n)}} B\left(x, 2 n^{-1}\right) \backslash\left(F_{0} \cup \bigcup_{i<n} E_{i}\right)$,

(E4) $B\left(x, 2 n^{-1}\right) \cap E_{n} \neq \emptyset$ for each $x \in F_{\lambda(n)} \backslash F_{\lambda(n)+1}$,

(E5) $\operatorname{cl} K \subset B(x, 2 \operatorname{dist}(x, K))$ for each $x \in F_{0}$ and each $K \in \mathcal{L}_{\varrho(n)}^{\lambda(n)}$,

(E6) for each $K \in \mathcal{L}_{\varrho(n)}^{\lambda(n)}$, there exists an $a_{K} \in F_{\lambda(n)} \backslash F_{\lambda(n)+1}$ such that $\operatorname{dist}\left(a_{K}, K\right)<n^{-1}$ and

$$
(\forall z \in K)\left|f\left(a_{K}\right)-f(z)\right|<n^{-1} \quad \text { or } \quad(\forall z \in K)|f(z)|>M_{n}\left(a_{K}\right),
$$

(E7) for each $\alpha<\xi$ and each $x \in F_{0} \backslash F_{\alpha}$, there exists an $r>0$ such that

$$
\lambda(n) \geq \alpha \Rightarrow B(x, r) \cap E_{n}=\emptyset .
$$

Fix an $n \in \mathbb{N}$ and assume that we have already defined the families $\mathcal{L}_{\varrho(i)}^{\lambda(i)}$ for all $i<n$. Put

$$
T_{n} \stackrel{\mathrm{df}}{=} \bigcup_{x \in F_{\lambda(n)} \backslash F_{\lambda(n)+1}} f^{-1}\left(U_{n}(x)\right) \cap B\left(x, n^{-1}\right) \backslash\left(F_{0} \cup \bigcup_{i<n} E_{i}\right) \subset \mathcal{e}_{f} .
$$

For each $t \in T_{n}$, choose $\varphi_{n}(t)>0$ so that

$$
\varphi_{n}(t)<\operatorname{dist}\left(t, F_{0} \cup \bigcup_{i<n} E_{i}\right) / 4 \text { and } \operatorname{diam} f\left[B\left(t, \varphi_{n}(t)\right)\right]<(2 n)^{-1} .
$$

Observe that for each $t \in T_{n}$,

$$
\varphi_{n}(t)<\operatorname{dist}\left(t, F_{0} \cup \bigcup_{i<n} E_{i}\right) / 4 \leq \operatorname{dist}\left(t, F_{\lambda(n)}\right) / 4<(4 n)^{-1} .
$$


Let $S_{n}$ be a maximal (with respect to inclusion) subset of $T_{n}$ with the property that

$$
d(x, y)>n^{-1} \text { whenever } x, y \in S_{n} \text { and } x \neq y .
$$

Define

$$
\mathcal{L}_{\varrho(n)}^{\lambda(n)} \stackrel{\text { df }}{=}\left\{B\left(t, \varphi_{n}(t)\right): t \in S_{n}\right\} .
$$

We shall verify that the family $\mathcal{L}_{\varrho(n)}^{\lambda(n)}$ fulfills $(\mathrm{E} 1)-(\mathrm{E} 7)$.

Let $t \in S_{n}$. Then by $(4), \operatorname{diam} B\left(t, \varphi_{n}(t)\right) \leq 2 \varphi_{n}(t)<(2 n)^{-1}$.

To prove (E2) fix an $x \in \operatorname{cl} E_{n}$. Let $\left(x_{k}\right) \subset E_{n}$ be convergent to $x$. For each $k$, choose a $t_{k} \in S_{n}$ such that $x_{k} \in \operatorname{cl} B\left(t_{k}, \varphi_{n}\left(t_{k}\right)\right)$. Observe that there is a $k_{0} \in \mathbb{N}$ such that for each $k>k_{0}$, by (4),

$$
d\left(t_{k}, t_{k+1}\right) \leq d\left(t_{k}, x_{k}\right)+d\left(x_{k}, x\right)+d\left(x, x_{k+1}\right)+d\left(x_{k+1}, t_{k+1}\right)<n^{-1} .
$$

So by (5), $t_{k}=t_{k_{0}}$ for $k>k_{0}$, and consequently, $x \in \operatorname{cl} B\left(t_{k_{0}}, \varphi_{n}\left(t_{k_{0}}\right)\right) \subset E_{n}$.

Let $t \in S_{n}$ and $z \in \operatorname{cl} B\left(t, \varphi_{n}(t)\right)$. Then $t \in T_{n}$, so by (4),

$$
\operatorname{dist}\left(z, F_{\lambda(n)}\right) \leq d(z, t)+\operatorname{dist}\left(t, F_{\lambda(n)}\right)<\varphi_{n}(t)+n^{-1}<2 n^{-1} .
$$

On the other hand,

$$
\operatorname{dist}\left(z, F_{0} \cup \bigcup_{i<n} E_{i}\right) \geq \operatorname{dist}\left(t, F_{0} \cup \bigcup_{i<n} E_{i}\right)-d(z, t)>3 \varphi_{n}(t)>0 .
$$

It follows that $z \in \bigcup_{x \in F_{\lambda(n)}} B\left(x, 2 n^{-1}\right) \backslash\left(F_{0} \cup \bigcup_{i<n} E_{i}\right)$.

Now let $x \in F_{\lambda(n)} \backslash F_{\lambda(n)+1}$. Take any $m>n$ with

$$
m^{-1} \leq \operatorname{dist}\left(x, \bigcup_{i<n} E_{i}\right) .
$$

By (1), there is a $z \in f^{-1}\left(U_{n}(x)\right) \cap B\left(x, m^{-1}\right) \backslash F_{0}$. Clearly $z \in T_{n}$. If $z \in S_{n}$, then we are done. So, assume that $z \notin S_{n}$. Then by the maximality of $S_{n}$, there exists a $t \in S_{n}$ with $d(z, t) \leq n^{-1}$, whence $t \in B\left(x, 2 n^{-1}\right) \cap E_{n}$.

Fix $x \in F_{0}$ and $K=B\left(t, \varphi_{n}(t)\right) \in \mathcal{L}_{\varrho(n)}^{\lambda(n)}$. Then

$$
\operatorname{dist}(x, K) \geq d(x, t)-\varphi_{n}(t) \geq \operatorname{dist}\left(t, F_{0}\right)-\varphi_{n}(t)>3 \varphi_{n}(t)>\operatorname{diam} K
$$

and

$$
\text { cl } K \subset \operatorname{cl} B(x, \operatorname{dist}(x, K)+\operatorname{diam} K) \subset B(x, 2 \operatorname{dist}(x, K)) .
$$


Take any $t \in S_{n}$ and $z \in K \stackrel{\mathrm{df}}{=} B\left(t, \varphi_{n}(t)\right)$. By definition, there is an $a_{K} \in F_{\lambda(n)} \backslash F_{\lambda(n)+1}$ such that $t \in f^{-1}\left(U_{n}\left(a_{K}\right)\right) \cap B\left(a_{K}, n^{-1}\right)$. Then clearly $\operatorname{dist}\left(a_{K}, K\right)<n^{-1}$. If $\left|f(t)-f\left(a_{K}\right)\right|<(2 n)^{-1}$, then by (3),

$$
\left|f\left(a_{K}\right)-f(z)\right| \leq\left|f\left(a_{K}\right)-f(t)\right|+\operatorname{diam} f[K]<n^{-1} .
$$

Otherwise $|f(t)|>2 M_{n}\left(a_{K}\right)>M_{n}\left(a_{K}\right)+n$. Using again (3), we obtain

$$
|f(z)| \geq|f(t)|-\operatorname{diam} f[K]>M_{n}\left(a_{K}\right) .
$$

Now fix $\alpha<\xi$ and $x \in F_{0} \backslash F_{\alpha}$. Then $\operatorname{dist}\left(x, F_{\alpha}\right)>4 m^{-1}$ for some $m \in \mathbb{N}$. By (E2) and (E3),

$$
V \stackrel{\text { df }}{=} B\left(x, 2 m^{-1}\right) \backslash \bigcup_{n<m} E_{n}
$$

is an open neighborhood of $x$. Choose an $r \in\left(0, m^{-1}\right)$ so that $B(x, r) \subset V$.

Finally fix an $n \in \mathbb{N}$ with $\lambda(n) \geq \alpha$ and take any $K \in \mathcal{L}_{\varrho(n)}^{\lambda(n)}$. If $n<m$, then by (6),

$$
B(x, r) \cap \operatorname{cl} K \subset V \cap E_{n}=\emptyset .
$$

In the opposite case by (E3),

$$
B(x, r) \cap \operatorname{cl} K \subset B(x, r) \cap \bigcup_{x \in F_{\alpha}} B\left(x, 2 m^{-1}\right)=\emptyset .
$$

\subsection{The Main Part of the Proof of the Theorem.}

First observe that

for each $t \in X \backslash F_{0}$, there exists an $r>0$ such that $\operatorname{cl} K \cap B(t, r) \neq \emptyset$

for at most one $K \in \bigcup_{n \in \mathbb{N}} \mathcal{L}_{\varrho(n)}^{\lambda(n)}$.

For this, fix a $t \in X \backslash F_{0}$. Then $\operatorname{dist}\left(t, F_{0}\right)>4 m^{-1}$ for some $m \in \mathbb{N}$. Observe that by (E3),

$$
\bigcup_{n \geq m} E_{n} \subset \bigcup_{n \geq m} \bigcup_{x \in F_{\lambda(n)}} B\left(x, 2 n^{-1}\right) \subset \bigcup_{n \geq m} \bigcup_{x \in F_{\lambda(n)}} B\left(x, 2 m^{-1}\right),
$$

whence $B\left(t, 2 m^{-1}\right) \cap \bigcup_{n \geq m} E_{n}=\emptyset$. By (E2), the set $\bigcup_{n<m} E_{n}$ is closed. So, if $t \notin \bigcup_{n \in \mathbb{N}} E_{n}$, then $B(t, r) \cap \bigcup_{n \in \mathbb{N}} E_{n}=\emptyset$ for some $r>0$. Otherwise since by (E3), the sets $E_{1}, E_{2}, \ldots$ are pairwise disjoint, there is a unique $k<m$ 
such that $t \in E_{k}$. By (5), there is a unique $K \in \mathcal{L}_{\varrho(k)}^{\lambda(k)}$ with $t \in \operatorname{cl} K$. Using again (5) we can find an $r>0$ such that

$$
B(t, r) \cap\left(\left(E_{k} \backslash K\right) \cup \bigcup_{n \neq k} E_{n}\right)=\emptyset .
$$

Observe also that by (E5), for each $x \in F_{0}$ and each $K \in \bigcup_{n \in \mathbb{N}} \mathcal{L}_{\varrho(n)}^{\lambda(n)}$,

$$
\operatorname{diam}(\{x\} \cup K) \leq \operatorname{diam} B(x, 2 \operatorname{dist}(x, K)) \leq 4 \operatorname{dist}(x, K) .
$$

The following notation is standard. For $x \in X$, we define

$$
f^{+}(x) \stackrel{\mathrm{df}}{=} \max \{f(x), 0\}, f^{-}(x) \stackrel{\mathrm{df}}{=} \max \{-f(x), 0\} .
$$

Define the functions $f_{1}, f_{2}: X \rightarrow \mathbb{R}$ as follows:

$$
\begin{aligned}
f_{1}(x) \stackrel{\text { df }}{=} \begin{cases}f^{+}(x)+h_{D}(x) & \text { if } x \in X \backslash D, \\
f^{+}(x)+h_{F_{\alpha+1}}(x) & \text { if } x \in F_{\alpha} \backslash F_{\alpha+1}, \alpha<\xi, \\
f^{+}\left(a_{K}\right)+h_{F_{\lambda(n)+1}}\left(a_{K}\right) & \text { if } x \in \operatorname{cl} K, K \in \mathcal{L}_{\varrho(n)}^{\lambda(n)}, \varrho(n) \text { even, } \\
f(x)+f^{-}\left(a_{K}\right)+h_{F_{\lambda(n)+1}}\left(a_{K}\right) & \text { if } x \in \operatorname{cl} K, K \in \mathcal{L}_{\varrho(n)}^{\lambda(n)}, \varrho(n) \text { odd, }\end{cases} \\
f_{2}(x) \stackrel{\text { df }}{=} \begin{cases}-f^{-}(x)-h_{D}(x) & \text { if } x \in X \backslash D, \\
-f^{-}(x)-h_{F_{\alpha+1}}(x) & \text { if } x \in F_{\alpha} \backslash F_{\alpha+1}, \alpha<\xi, \\
f(x)-f^{+}\left(a_{K}\right)-h_{F_{\lambda(n)+1}}\left(a_{K}\right) & \text { if } x \in \operatorname{cl} K, K \in \mathcal{L}_{\varrho(n)}^{\lambda(n)}, \varrho(n) \text { even, } \\
-f^{-}\left(a_{K}\right)-h_{F_{\lambda(n)+1}}\left(a_{K}\right) & \text { if } x \in \operatorname{cl} K, K \in \mathcal{L}_{\varrho(n)}^{\lambda(n)}, \varrho(n) \text { odd, }\end{cases}
\end{aligned}
$$

where

$$
D \stackrel{\text { df }}{=} F_{0} \cup \bigcup_{n \in \mathbb{N}} \bigcup_{K \in \mathcal{L}_{\varrho(n)}^{\lambda(n)}} \operatorname{cl} K=F_{0} \cup \bigcup_{n \in \mathbb{N}} E_{n} .
$$

Observe that $D$ is closed.

Indeed, let $\left(t_{k}\right) \subset D$ be convergent to some $t_{0} \in X$. If there is a subsequence $\left(t_{k_{m}}\right) \subset F_{0}$, then $t_{0} \in \operatorname{cl} F_{0} \subset D$.

So, assume that for each $k$, there is an $n_{k} \in \mathbb{N}$ such that $t_{k} \in E_{n_{k}}$. If $n_{k}=n_{0}$ for infinitely many $k$, then by (E2), $t_{0} \in E_{n_{0}} \subset D$.

Finally if $n_{k} \rightarrow \infty$, then by (E3), for each $k$, there is an $x_{k} \in F_{\lambda\left(n_{k}\right)} \subset F_{0}$ such that $t_{k} \in B\left(x_{k}, 2 n_{k}^{-1}\right)$. Consequently,

$$
d\left(x_{k}, t_{0}\right) \leq d\left(x_{k}, t_{k}\right)+d\left(t_{k}, t_{0}\right) \leq 2 n_{k}^{-1}+d\left(t_{k}, t_{0}\right) \rightarrow 0
$$

and $t_{0} \in \operatorname{cl} F_{0} \subset D$.

Clearly $f=f_{1}+f_{2}$ on $X$. We shall verify that $f_{1}$ is quasi-continuous and its graph is closed. The proofs of the analogous statements for $f_{2}$ are similar, we shall omit them. 


\subsection{1 $f_{1}$ is Quasi-continuous.}

Fix an $x \in X$, a neighborhood $U$ of $x$, and an $\varepsilon \in(0,1)$. We consider several cases.

If $x \notin D$, then $f_{1}$ is continuous at $x$. So, the set

$$
V \stackrel{\mathrm{df}}{=} U \cap f_{1}^{-1}\left(\left(f_{1}(x)-\varepsilon / 3, f_{1}(x)+\varepsilon / 3\right)\right)
$$

is an open neighborhood of $x$ contained in $U$ such that $\operatorname{diam} f_{1}[V]<\varepsilon$.

If $x \in D \backslash F_{0}$, then there is an $n \in \mathbb{N}$ and a $K \in \mathcal{L}_{\varrho(n)}^{\lambda(n)}$ with $x \in \operatorname{cl} K$. Since $f_{1} \uparrow_{\mathrm{cl} K}$ is continuous, the set

$$
V \stackrel{\text { df }}{=} U \cap K \cap f_{1}^{-1}\left(\left(f_{1}(x)-\varepsilon / 3, f_{1}(x)+\varepsilon / 3\right)\right)
$$

is a nonvoid and open subset of $U$ such that $\operatorname{diam} f_{1}[V \cup\{x\}]<\varepsilon$.

Finally assume that $x \in F_{\alpha} \backslash F_{\alpha+1}$ for some $\alpha<\xi$. Since $f \uparrow_{F_{\alpha} \backslash F_{\alpha+1}}$ is continuous, we can choose an $r \in(0,1)$ such that $B(x, 3 r) \subset U \backslash F_{\alpha+1}$ and $\operatorname{diam} f\left[B(x, 2 r) \cap F_{\alpha}\right]<\varepsilon / 2$. Let $n>6 r^{-2} \varepsilon^{-1}$ be such that $\varrho(n)$ is even and $\lambda(n)=\alpha$. By $(\mathrm{E} 4)$, there is a $K \in \mathcal{L}_{\varrho(n)}^{\lambda(n)}$ such that $V \stackrel{\text { df }}{=} B\left(x, 2 n^{-1}\right) \cap K \neq \emptyset$. Notice that $V$ is a nonvoid open subset of $U$. Choose $a_{K} \in F_{\alpha} \backslash F_{\alpha+1}$ according to (E6). Then by (8),

$$
d\left(x, a_{K}\right) \leq \operatorname{diam}(\{x\} \cup K)+\operatorname{dist}\left(a_{K}, K\right) \leq 4 \operatorname{dist}(x, K)+n^{-1}<9 n^{-1}<2 r .
$$

Hence $\operatorname{dist}\left(a_{K}, F_{\alpha+1}\right) \geq \operatorname{dist}\left(x, F_{\alpha+1}\right)-d\left(x, a_{K}\right)>r$ and

$$
\begin{aligned}
\operatorname{diam} f_{1}[V \cup\{x\}] & =\left|f^{+}\left(a_{K}\right)+h_{F_{\lambda(n)+1}}\left(a_{K}\right)-f^{+}(x)-h_{F_{\alpha+1}}(x)\right| \\
& \leq\left|f^{+}\left(a_{K}\right)-f^{+}(x)\right|+\left|h_{F_{\alpha+1}}\left(a_{K}\right)-h_{F_{\alpha+1}}(x)\right| \\
& <\varepsilon / 2+\frac{\left|\operatorname{dist}\left(x, F_{\alpha+1}\right)-\operatorname{dist}\left(a_{K}, F_{\alpha+1}\right)\right|}{\operatorname{dist}\left(a_{K}, F_{\alpha+1}\right) \operatorname{dist}\left(x, F_{\alpha+1}\right)} \\
& <\varepsilon / 2+\frac{d\left(x, a_{K}\right)}{3 r^{2}}<\varepsilon / 2+\frac{3}{n r^{2}}<\varepsilon .
\end{aligned}
$$

\subsubsection{The Graph of $f_{1}$ is Closed.}

Now take any point $\langle x, y\rangle$ from the closure of the graph of $f_{1}$. Let $\left(x_{k}\right) \subset X$ be such that $x_{k} \rightarrow x$ and $f_{1}\left(x_{k}\right) \rightarrow y$. We shall prove that $y=f_{1}(x)$. We consider several cases.

- If there is a subsequence $\left(x_{k_{m}}\right) \subset F_{0}$, then $x \in F_{0}$, whence $x \in F_{\alpha} \backslash F_{\alpha+1}$ for some $\alpha<\xi$. For each $m \in \mathbb{N}$, there is a unique $\alpha_{m}<\xi$ such that $x_{k_{m}} \in F_{\alpha_{m}} \backslash F_{\alpha_{m}+1}$. Since $F_{\alpha+1}$ is closed, $\alpha_{m} \leq \alpha$ for sufficiently big $m$. 
Notice that $f_{1} \geq 0$ on $F_{0}$, whence $y \geq 0$. For sufficiently big $m$, we have $d\left(x_{k_{m}}, x\right)<(y+1)^{-1}$ and $f_{1}\left(x_{k_{m}}\right)<y+1$. Then $\operatorname{dist}\left(x_{k_{m}}, F_{\alpha_{m}+1}\right)=$ $\frac{1}{h_{F_{\alpha_{m}+1}}\left(x_{k_{m}}\right)} \geq \frac{1}{f_{1}\left(x_{k_{m}}\right)}>\frac{1}{y+1}>d\left(x_{k_{m}}, x\right)$, whence $x \notin F_{\alpha_{m}+1}$ and $\alpha_{m}=$ $\alpha$. Since $f \uparrow_{F_{\alpha} \backslash F_{\alpha+1}}$ is continuous,

$y=\lim _{k \rightarrow \infty} f_{1}\left(x_{k}\right)=\lim _{m \rightarrow \infty}\left(f^{+}\left(x_{k_{m}}\right)+h_{F_{\alpha+1}}\left(x_{k_{m}}\right)\right)=f^{+}(x)+h_{F_{\alpha+1}}(x)=f_{1}(x)$.

- If there is a subsequence $\left(x_{k_{m}}\right) \subset X \backslash D$, then $\limsup _{m \rightarrow \infty} h_{D}\left(x_{k_{m}}\right) \leq$ $\lim _{m \rightarrow \infty} f_{1}\left(x_{k_{m}}\right)=y<\infty$. Hence $x=\lim _{m \rightarrow \infty} x_{k_{m}} \in X \backslash D \subset \mathcal{C}_{f_{1}}$ and $y=f_{1}(x)$.

- Finally assume that $\left(x_{k}\right) \subset D \backslash F_{0}$. For each $k$, there are unique $n_{k} \in \mathbb{N}$ and $K_{k} \in \mathcal{L}_{\varrho\left(n_{k}\right)}^{\lambda\left(n_{k}\right)}$ such that $x_{k} \in K_{k}$. If $x \notin F_{0}$, then by (7), there is an $n \in \mathbb{N}$ and a $K \in \mathcal{L}_{\varrho(n)}^{\lambda(n)}$ such that $K_{k}=K$ for sufficiently big $k$. By (E2), $f_{1} \uparrow_{\mathrm{cl} K}$ is continuous, whence $y=f_{1}(x)$. Now assume that $x \in F_{\alpha} \backslash F_{\alpha+1}$ for some $\alpha<\xi$. Use (E7) to find an $r \in\left(0,(2|y|+1)^{-1}\right)$ such that

$$
(\forall n \in \mathbb{N})\left(\lambda(n)>\alpha \Rightarrow B(x, r) \cap E_{n}=\emptyset\right) .
$$

For each $k$, choose $a_{K_{k}}$ according to (E6). Notice that by (E2), there is no constant subsequence of $\left(n_{k}\right)$. Consequently, $n_{k} \rightarrow \infty$ and by (8),

$$
d\left(x, a_{K_{k}}\right) \leq \operatorname{diam}\left(\{x\} \cup K_{k}\right)+\operatorname{dist}\left(a_{K_{k}}, K_{k}\right) \leq 4 d\left(x, x_{k}\right)+n_{k}^{-1} \rightarrow 0 .
$$

So, there is a $k_{0} \in \mathbb{N}$ such that

$$
d\left(x_{k}, x\right)<r \quad \text { and } \quad\left|f_{1}\left(x_{k}\right)\right|<|y|+2^{-1}<n_{k} \quad \text { for } k>k_{0} .
$$

By (9), $\lambda\left(n_{k}\right) \leq \alpha$ for each $k>k_{0}$. We consider two subcases.

( Assume that there is a subsequence $\left(n_{k_{m}}\right)$ such that $\varrho\left(n_{k_{m}}\right)$ is even for each $m$. For sufficiently big $m$, we have $k_{m}>k_{0}$ and $n_{k_{m}}>2|y|+1$. Then

$$
\begin{aligned}
d\left(x_{k_{m}}, F_{\lambda\left(n_{k_{m}}\right)+1}\right) & \geq d\left(a_{K_{k_{m}}}, F_{\lambda\left(n_{k_{m}}\right)+1}\right)-d\left(x_{k_{m}}, a_{k_{m}}\right) \\
& >\frac{1}{f_{1}\left(x_{k_{m}}\right)}-n_{k_{m}}^{-1}>\frac{1}{2|y|+1}>d\left(x_{k_{m}}, x\right),
\end{aligned}
$$

whence $x \notin F_{\lambda\left(n_{k_{m}}\right)+1}$ and by (9), $\lambda\left(n_{k_{m}}\right)=\alpha$. Since $f \uparrow_{F_{\alpha} \backslash F_{\alpha+1}}$ is continuous, $y=\lim _{m \rightarrow \infty}\left(f^{+}\left(x_{k_{m}}\right)+h_{F_{\lambda\left(n_{k_{m}}\right)+1}}\left(x_{k_{m}}\right)\right)=f^{+}(x)+h_{F_{\alpha+1}}(x)=f_{1}(x)$.

\ So, assume that $\varrho\left(n_{k}\right)$ is odd for each $k$. Then for each $k>k_{0}$, by (11),

$$
\begin{aligned}
\left|f\left(x_{k}\right)\right| & \leq\left|f\left(x_{k}\right)+f^{-}\left(a_{K_{k}}\right)+h_{F_{\lambda\left(n_{k}\right)+1}}\left(a_{K_{k}}\right)\right|+\left|f^{-}\left(a_{K_{k}}\right)\right|+h_{F_{\lambda\left(n_{k}\right)+1}}\left(a_{K_{k}}\right) \\
& =\left|f_{1}\left(x_{k}\right)\right|+\left|f^{-}\left(a_{K_{k}}\right)\right|+h_{F_{\lambda\left(n_{k}\right)+1}}\left(a_{K_{k}}\right)<M_{n_{k}}\left(a_{K_{k}}\right),
\end{aligned}
$$


whence by (E6),

$$
\left|f\left(a_{K_{k}}\right)-f\left(x_{k}\right)\right|<n_{k}^{-1}
$$

It follows that

$$
\begin{aligned}
|y|+2^{-1} & >f_{1}\left(x_{k}\right)=f\left(x_{k}\right)+f^{-}\left(a_{K_{k}}\right)+h_{F_{\lambda\left(n_{k}\right)+1}}\left(a_{K_{k}}\right) \\
& \geq h_{F_{\lambda\left(n_{k}\right)+1}}\left(a_{K_{k}}\right)-\left|f\left(x_{k}\right)-f\left(a_{K_{k}}\right)\right|+\left(f\left(a_{K_{k}}\right)+f^{-}\left(a_{K_{k}}\right)\right) \\
& >h_{F_{\lambda\left(n_{k}\right)+1}}\left(a_{K_{k}}\right)-n_{k}^{-1} .
\end{aligned}
$$

By (10), we conclude that $x \notin F_{\lambda\left(n_{k}\right)+1}$, and by $(9)$, that $\lambda\left(n_{k}\right)=\alpha$. Recall that $f^{+} \uparrow_{F_{\alpha} \backslash F_{\alpha+1}}$ is continuous. So by (12) and (10),

$$
\begin{aligned}
\left|y-f_{1}(x)\right| & =\lim _{k \rightarrow \infty}\left|f_{1}\left(x_{k}\right)-f_{1}(x)\right| \\
= & \lim _{k \rightarrow \infty}\left|f\left(x_{k}\right)+f^{-}\left(a_{K_{k}}\right)+h_{F_{\lambda\left(n_{k}\right)+1}}\left(a_{K_{k}}\right)-f^{+}(x)-h_{F_{\alpha+1}}(x)\right| \\
\leq & \lim _{k \rightarrow \infty}\left|f\left(x_{k}\right)-f\left(a_{K_{k}}\right)\right|+\lim _{k \rightarrow \infty}\left|f^{+}\left(a_{K_{k}}\right)-f^{+}(x)\right| \\
& \quad+\lim _{k \rightarrow \infty}\left|h_{F_{\alpha+1}}\left(a_{K_{k}}\right)-h_{F_{\alpha+1}}(x)\right|=0 .
\end{aligned}
$$

This completes the proof.

\section{References}

[1] J. Borsík, Sums of quasicontinuous functions defined on pseudometrizable spaces, Real Anal. Exch., 22(1) (1996), 328-337.

[2] J. Borsík, Sums, differences, products and quotients of closed graph functions, Tatra Mt. Math. Publ., 24(2) (2002), 117-123.

[3] J. Borsík, J. Doboš, and M. Repický, Sums of quasicontinuous functions with closed graphs, Real Anal. Exch., 25(2) (2000), 679-690.

[4] S. Kempisty, Sur les fonctions quasicontinues, Fund. Math., 19 (1932), 184-197.

[5] T. Neubrunn, Quasi-continuity, Real Anal. Exch., 14(2) (1988-89), 259306.

[6] R. J. O'Malley, Baire* 1, Darboux functions, Proc. Amer. Math. Soc., 60 (1976), 187-192.

[7] D.E. Peek, Characterizations of Baire* 1 functions in general settings, Proc. Amer. Math. Soc., 95 (1985), 557-580. 